\title{
An improved method/setup for extended testing after the Cigre, Method II
}

\author{
Henriksen, Mogens; Holbøll, Joachim; Rygal, Roman
}

Published in:

I E E E International Symposium on Electrical Insulation. Conference Record

Link to article, DOI:

10.1109/ELINSL.1992.247033

Publication date:

1992

Document Version

Publisher's PDF, also known as Version of record

Link back to DTU Orbit

Citation (APA):

Henriksen, M., Holbøll, J., \& Rygal, R. (1992). An improved method/setup for extended testing after the Cigre, Method II. I E E E International Symposium on Electrical Insulation. Conference Record, 144-148.

https://doi.org/10.1109/ELINSL.1992.247033

\section{General rights}

Copyright and moral rights for the publications made accessible in the public portal are retained by the authors and/or other copyright owners and it is a condition of accessing publications that users recognise and abide by the legal requirements associated with these rights.

- Users may download and print one copy of any publication from the public portal for the purpose of private study or research.

- You may not further distribute the material or use it for any profit-making activity or commercial gain

- You may freely distribute the URL identifying the publication in the public portal 


\section{AN IMPROVED METHOD/SETUP FOR EXTENDED TESTING AFTER THE CIGRÉ, METHOD II.}

\author{
M. Henriksen, J.T. Holbøll \\ Electric Power Engineering Department \\ Technical University of Denmark \\ Building 325, DK-2800 Lyngby \\ DENMARK
}

\author{
Roman Rygal \\ Department of Electrical Engineering \\ Technical University of Czestochowa \\ PL-42-201 Czestochowa \\ POLAND
}

\section{Abstract}

This paper deals with the description of an improved version of the Cigré, Method II, test method used for testing the resistance of insulating materials to partial discharges.

Applying test data acquisition instrumentation in combination with individual test cells to the Cigré, Method II allows for each sample automatic gain control to obtain maximum signal to noise ratio, apparent peak recording and pulse phase analyses (PPA). It has been found that parameters such as selective fuse protecting of the different test objects, the sample production and preparation etc. are of the utmost importance for the end result of this test.

The paper also contains results obtained using the new system on epoxy with and without fillers, and, based on these, a discussion of the critical points of the method. It will also briefly be discussed if the results obtained using this new improved test method can be compared to a test method applying samples containing one single void in said materials.

\section{$\underline{\text { Introduction }}$}

A system for testing insulating materials for their resistance against electrical discharges and its application will be described. The system is based on the Cigré, Method II as described by CIGRÉ WG 15-06 [1], where the material is exposed to partial discharges formed in a strong, inhomogeneous field in a flat cylindrical void. Accelerated PD-aging of the material after said method allows for measuring the lifetime of different materials within practical time limits (normally $<1000$ hours). A number of specimens can be exposed to the same voltage, but in the case of breakdown, tripping out of the failed specimen will be necessary. Measurements of the apparent charge on the electrodes as a function of aging time have shown that a voltage outage of several hours, due to breakdown in one specimen, leads to considerable and permanent changes in the character of the discharges in the other specimens [2]. For that reason a system has been developed, which is based on individual fuse protected test cells, with a voltage outage of no more than 5 seconds after an individual test sample has broken down.

Earlier measured lifetimes showed high scatter and sometimes very short lifetimes, especially in connection with filled materials, which hardly could be explained. In order to find the causes of this problem in the normal Cigré, Method II, the system was extended to continuous PD measurements and pulse analyses, in order to give some more detailed information about the exposure of the material under test, so as to be able to exclude some of the most extreme results from the statistical treatment.

The extended system has been used for investigations of the PDresistance of epoxy plastic with different fillers, the results of which are also described and discussed in the following.

\section{Test Setup}

The Cigré, Method II, electrode configuration is shown in fig.1.

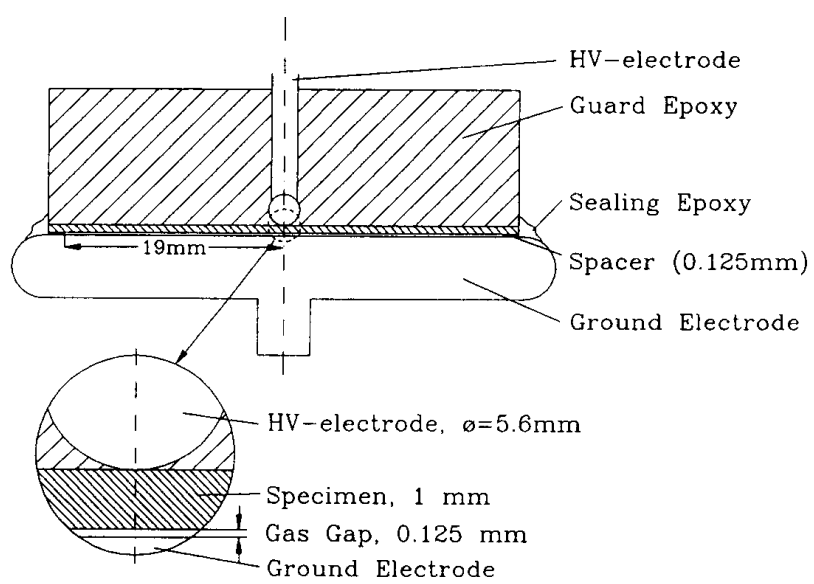

Fig.1: Cigré, Method II, Electrode configuration.

A $1 \mathrm{~mm}$ plate of the test material is glued directly onto the polished underside of the high voltage electrode, formed by a $5.6 \mathrm{~mm}$ sphere cast into epoxy. A flat cavity is formed by the underside of the material to be tested and the metal ground electrode, separated by a $0.125 \mathrm{~mm}$ high kapton ring. This configuration ensures a strong, inhomogeneous field in the cavity, hereby concentrating the discharges in an area with a radius of about twice that of the sphere. The test voltage is $30 \mathrm{kV}_{\mathrm{rms}}, 50 \mathrm{~Hz}$, under which exposure the lifetime for the different materials is measured.

As shown in fig.2, the extended system is based on individual test cells, each containing a high voltage fuse and the electrode configuration as shown in fig.1, plus an RLC-circuit as measuring impedance.

The high voltage fuses consist of a $10 \mu \mathrm{m}$ silver wire taken from a $50 \mathrm{~mA}$ fuse and extended with a long $0.1 \mathrm{~mm}$ copper wire. To prevent corona, two separated cylindrical shields enclose the wire. In the case of breakdown in a sample the thin silver wire blows and the copper wire will slide down and short the lower shield and the high voltage electrode to ground. With the rest of the fuse wire covered by the upper shield and the specimen grounded, neither corona nor discharges in the specimen occur, in order to avoid disturbance of the PD measurements on the intact specimens. When a breakdown occurs, the overcurrent protection of the high voltage transformer turns off the test voltage, whereupon it is re-established automatically after 5 seconds. 


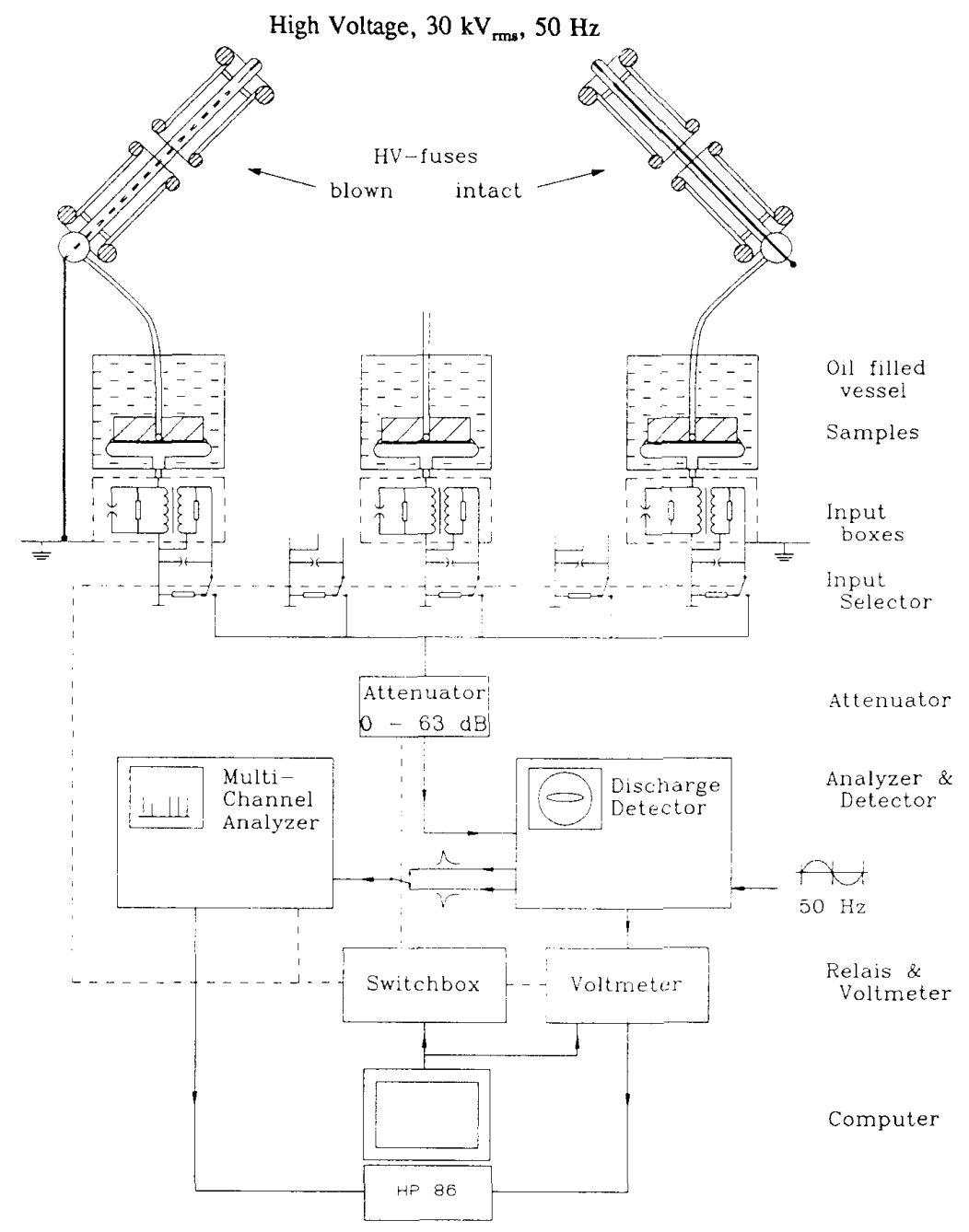

Fig.2: Measuring system

$\underline{\text { Results }}$

PD detection is based on in-line measurements of the discharge current through a measuring impedance formed by an RLC circuit as shown in fig.2. Via the input selector, the discharge pulse is sent to an ERA discharge detector, with a bandwidth of $10-300 \mathrm{kHz}$ in connection with the RLC circuit. To obtain maximum signal to noise ratio, the gain is automatically optimized for each sample by an attenuator/amplifier combination at the input of the discharge detector. The detector shows phase as well as height of the discharge pulses and has the maximum discharge pulse as a readable output. At fixed intervals, pulse phase analyses were performed by a computer controlled analyzer.

Input control, optimizing the gain, peak charge reading and analyzer control is automatically performed by an HP 86 computer.

A detailed description of the extended system is found in [3]
The extended system has been used for investigations of the resistance of unfilled and filled epoxies to electrical discharges. The epoxy used for the tests was a standard type Bisphenol-A Diglycidylether cured with an acidanhydride curing agent containing $60 \%$ (weight) filler. The used filler types were dolomite- $\mathrm{CaCO}_{3} \cdot \mathrm{MgCO}_{3}$ (5 samples), alumina- $\mathrm{Al}_{2} \mathrm{O}_{3}$ (10 samples) and silica- $\mathrm{SiO}_{2}$ (5 samples).

\section{Surface Investigations}

In order to obtain reproducible results the $1 \mathrm{~mm}$ thick test sample of the material to be tested must be homogeneous, without defects or impurities and with a naturally formed surface. With the used casting method, a pure and homogeneous material was obtained. But when casting the plate with a large contact area to a mold, as done in this case between steel plates, the surface structure of the mold will be imprinted on the sample surface as can be seen in fig. 3 . 


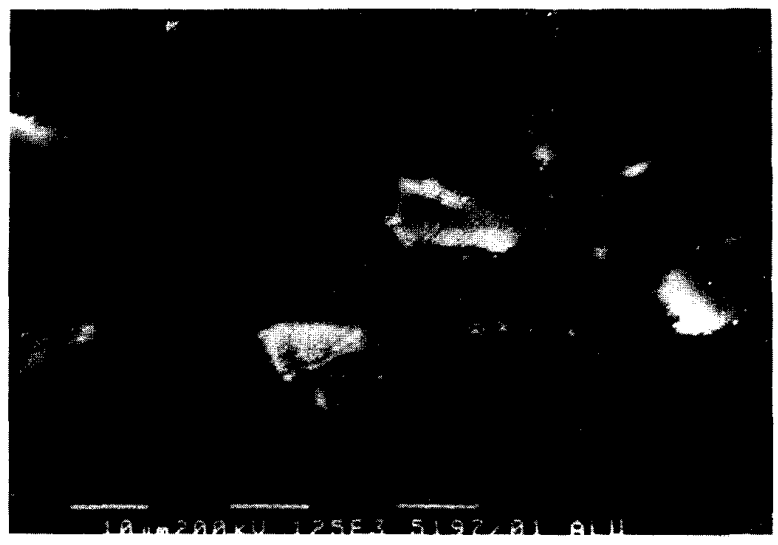

Fig.3: Surface of alumina filled epoxy before discharges.

Moreover, the release agent seemed to cause some irregularities on the surface.

After discharges, it was found that the surfaces had strongly deteriorated directly under the HV electrode and the epoxy had been removed from the surface of the upper filler particles. It was also found that the dolomite grains were partly decomposed by the discharges, whereas alumina and silica fillers did not show any deterioration. In some cases, the particles seemed to concentrate the discharge activity around the grains as shown in fig. 4 .

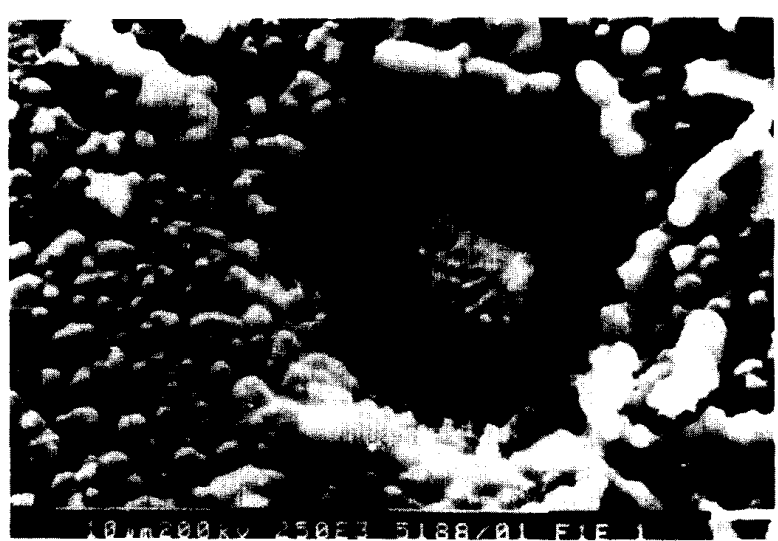

Fig.4: Surface of alumina filled epoxy after 50 hours with discharges.

\section{Discharge sequences}

During the first few hours of testing all the samples showed discharge sequences on the detector with few discharges on both the positive and the negative going halfcycle of the high voltage. After some hours the image changed to few discrete discharges in the beginning of each sequence, followed by a large number of very small discharge pulses as seen in fig. 5 .
Apart from somewhat higher discrete pulses on the negative going halfcycle, no asymmetry could be found between the two polarities. The magnitude difference of the two types of pulses was very large, some $20-40 \mathrm{~dB}$. As a consequence of this wide range and the limited dynamic range of the system, the discharge activity could not be determined satisfactorily.

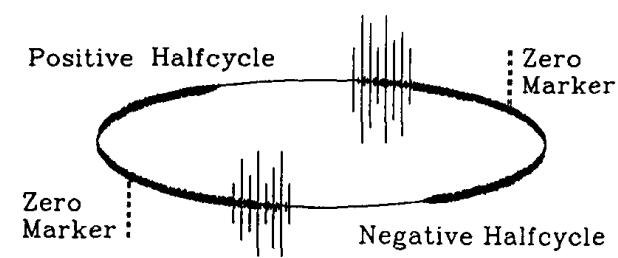

Fig.5: Discharge sequences as seen on the discharge detector after some hours aging of the material.

\section{Lifetime}

As can be seen in fig.6, the lifetime of the filled materials was characterized by high scatter with a maximum at 20,90 and more than 260 hours for dolomite, alumina and silica, respectively. The lifetime of unfilled epoxy was concentrated at about 70 hours.

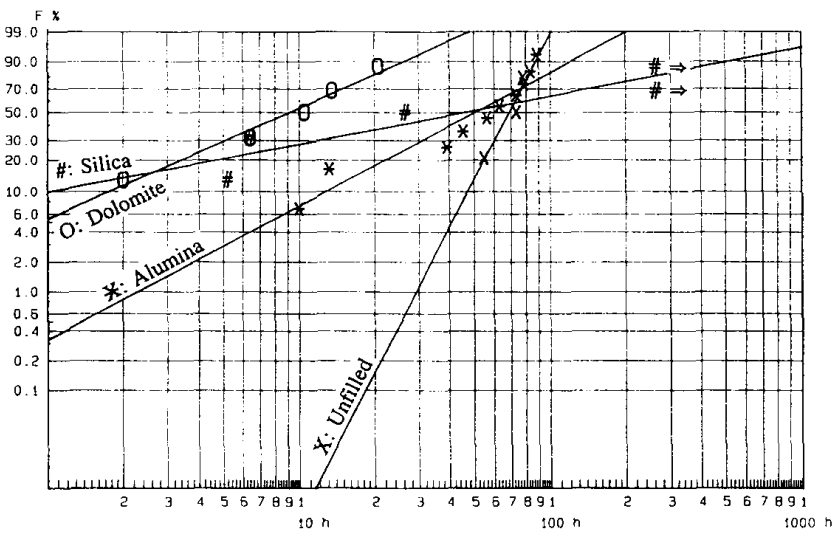

Fig.6: Lifetime for filled and unfilled epoxy when exposed for partial discharges after Cigré, Method II.

\section{Peak charge}

The measured peak charge as a function of time did not show any typical changes under aging, but before the breakdown, a unique decrease and increase in the peak charge can be observed in fig.7 and fig.8. Breakdown is illustrated by the sudden decrease of the peak charge. 


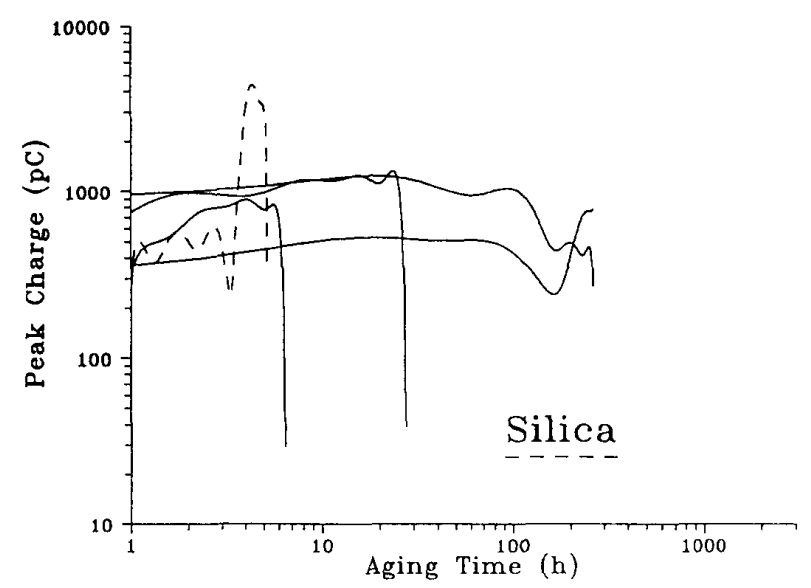

Fig.7: Peak charge as a function of aging time for silica filled epoxy.

In one of the silica samples the peak charge increased after 4 hours with a factor 10. After breakdown, microscopic investigations of this sample showed detachment of the high voltage electrode from the surrounding epoxy material being tested.

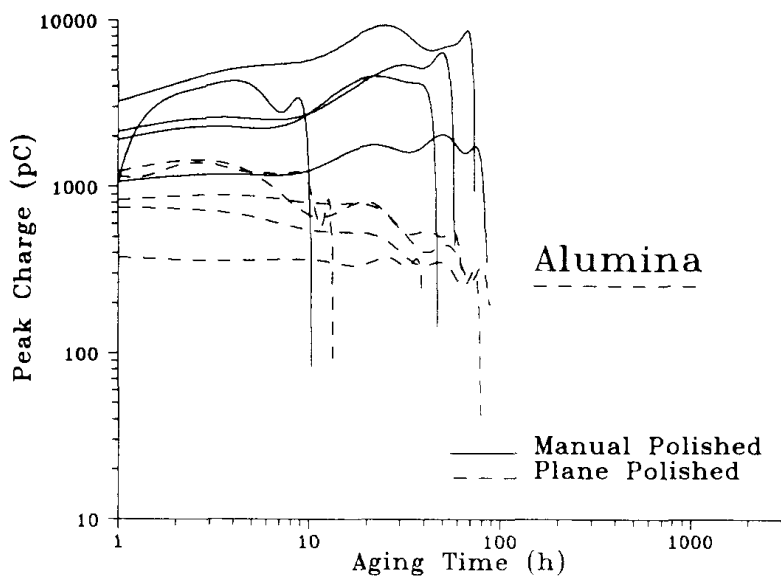

Fig.8: Peak charge as a function of aging time for alumina filled epoxy, with the HV-electrode being prepared in two different ways.

In fig. 8 results for alumina filled epoxy can be seen, where the under side of the high voltage electrodes was a) manually polished and b) almost plane polished. As can be seen, the slightly spherical shape of the manual polished underside of the electrodes resulted not only in a lower peak charge but also in a different development of the peak charge with time.

Furthermore, the diagrams show that a power break of 5 seconds after breakdown of a sample was without influence to the other samples.

\section{Pulse phase analyses}

The pulse phase analyses performed showed typically a phase distribution as shown in fig.9. The number of pulses is presented relatively, due to the limited dynamic range of the measuring system.

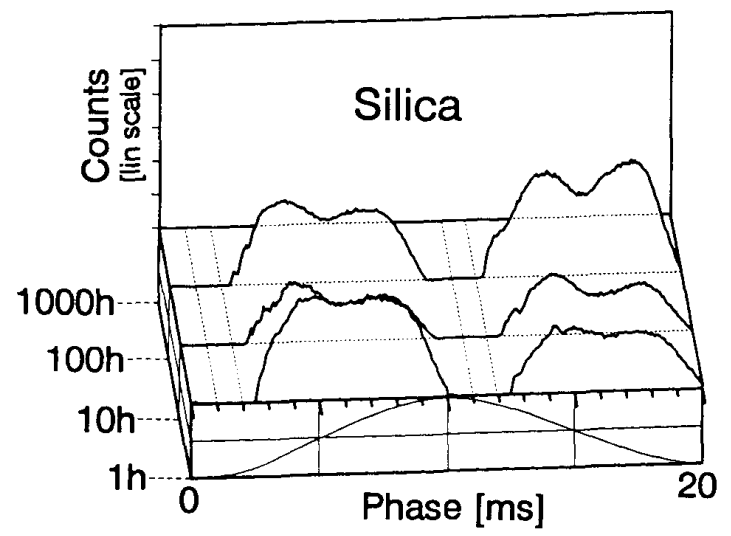

Fig.9: Phase distribution as a function of aging time

The phase distribution corresponds rather well to the observed image on the discharge detector. No particular changes of the distribution with time could be observed.

Regarding the peak charge shown in fig. $7 \& 8$ and the phase distributions in fig. 9 , these vary considerably from those found on the same material combinations when containing a single spherical void and tested under rather homogeneous field conditions [4]. The reason for this is, at the present, not clear.

\section{Discussion}

The Cigré, Method II as it exists today is a simple method but, based on the above results, it seems to lack -

1) measuring equipment to estimate the exposure of the material to discharges. Due to the difference between the two types of discharges in the highly stressed gap, a wide dynamic range of the measuring system is needed perhaps by use of a logarithmic amplifier. With a system being able to detect all pulses, pulse height analyses can be performed and the exposure of each specimen to discharges can be estimated and related to the lifetime. Moreover, as seen with the existing system, pulse analyses give the capability of excluding some extreme data, as caused by defects in the sample.

2) some detailed description of suggestions for methods to be used to produce samples of the electrical insulation materials to be tested, e.g. unfilled, filled or fiber reinforced epoxies etc. The used casting method with contact to a mould seems to cause weak points in the surface. Another question is, how perfect this surface has to be for not causing the observed short lifetimes when stressed as high as at $30 \mathrm{kV}$ test voltage. 
3) a much more detailed description of how the test cell is manufactured and put together. Due to the very small gap, the discharge character and consequently the deterioration processes seemed to be very sensitive to deviations in the $0.125 \mathrm{~mm}$ gap. It is the authors' experience that only very skilled personnel are able to obtain reproducible results.

Even if the exposure of the materials to discharges is not totally known, the significant differences between the lifetimes allows for a rough estimate of the resistance of the materials to electrical discharges. Exposed after the Cigré, Method II, dolomite decomposed and consequently had the shortest lifetime. The lifetimes of alumina and silica filled epoxy showed very high scatter and few breakdowns very soon after aging start. Microscopic investigations showed that some of these short lifetimes were caused by defects in the sample and therefore not should be included in the calculation of the PD resistance. Silica was the only material able to increase the lifetime above that of unfilled epoxy. The reason for this behaviour is not known, and further investigations have been started.

As described in [4] the same materials have been used in PD-aging tests in spherical voids under realistic conditions. A direct comparison between the results does not seem to be possible since the exposure to discharges under the Cigre, Method II is so high that other deterioration processes occur, as confirmed by the decomposition of dolomite grains. The different exposure to discharges is confirmed by the very different development of the pulse analyses. On the other hand, the strong exposure seems to give results that might be a supplement to aging phenomena under realistic conditions and, in that way, a wider range of information can be obtained, about the resistance of the material to electrical discharges.

\section{Conclusion}

The thoughts behind the Cigré, Method II test method to test the resistance of electrical insulation materials to partial discharges have been to develop a simple method to serve this purpose.

Having worked with this method for quite some time, we have ended up with an extended method in which we have some confidence, but it does require good technical testing expertise plus some advanced test equipment. We find these requirements to be essential for obtaining consistent test results.
The total test set-up used for these tests was very different from what is suggested in the Cigré, Method II, and the continuous observation of the development of the discharge magnitude and the discharge activity is looked upon as one of the means to improve the test procedure of the Cigre, Method II. Furthermore, it seems that there are three areas that need some attention:

a) The test voltage. The decomposition of dolomite grains indicates that $30 \mathrm{kV}$ might be too high.

b) The surface condition. Very short lifetimes of some test specimens might be caused by surface defects.

c) The electrode gap in the assembled electrode configuration is dependent of the final thickness of the kapton spacer and the accuracy by which the HV electrode can be made.

An application of the system to filled and unfilled epoxy plastic showed a typical behavior of the discharges and lifetimes strongly dependent of the filler type. In some cases, peak charge recording allowed for identifying abnormal exposure of the materials due to defects in the sample. That means that the combination of electrical test results combined with an observation of the occurred damage to the materials being tested has been found to be of great help in evaluating the test data.

\section{$\underline{\text { References }}$}

[1] W.R. Kodoll, H.C. Kärner, T. Tanaka, M. Ieda. "Internal Partial Discharge Testing." Cigré Paper 15-04. Paris, 1988.

[2] R. Rygal, M. Henriksen, J.T. Holbøll, E. Larsen. "Description of an Improved Cigré, Method II, Test Setup." Nordic Insulation Symposium. Västerås, 1992.

[3] K.G. Nielsen. "Surface Changes of Epoxy Plastic caused by Electrical Discharges." Master Thesis (in Danish). Electric Power Engineering Department, Technical University of Denmark. Lyngby, 1990.

[4] J.T. Holbøll, M. Henriksen. "Partial Discharge Patterns and Surface Deterioration in Voids in Filled and Unfilled Epoxy". IEEE International Symposium on Electrical Insulation, Baltimore, 1992. 